\title{
ESTADO NUTRICIONAL E PERCEPÇÃO CORPORAL ENTRE ADOLESCENTES DE UMA ESCOLA DO MUNICÍPIO DE GUARAPUAVA, PR
}

\author{
Aline Carla Chagas do AMARAL ${ }^{1}$ \\ Beatriz Valle GALEGO ${ }^{1}$ \\ Daiana NOVELLO ${ }^{2}$
}

\begin{abstract}
${ }^{1}$ Nutricionista, Universidade Estadual do Centro-Oeste (UNICENTRO).
${ }^{2}$ Professora, Doutora, Departamento de Nutrição (UNICENTRO). *E-mail: nutridai@gmail.com.
\end{abstract}

Recebido em: 15/08/2015 - Aprovado em: 08/01/2016 - Disponibilizado em: 30/07/2016

\begin{abstract}
Resumo: O objetivo do presente estudo foi avaliar o estado nutricional e a percepção corporal de adolescentes de uma escola municipal do município de Guarapuava, PR. O estudo foi realizado em uma escola municipal de Guarapuava, PR, participaram 39 adolescentes, de ambos os gêneros, com idade entre 10 e 16 anos. Foram aferidos peso e altura para avaliação do estado nutricional pelo Índice de Massa Corporal (IMC) e, posteriormente foi aplicado o questionário Body Shape Questionnaire (BSQ) para verificação da percepção corporal. Para avaliar a satisfação corporal dos alunos foi utilizado o Silhouette Matching Task (SMT). Os resultados mostraram que 79,5\% dos adolescentes estavam eutróficos, $5,1 \%$ obesos e 15,4\% em sobrepeso. Em geral, os indivíduos tiveram uma percepção positiva de sua imagem corporal, entretanto verificou-se uma prevalência de IMC com sobrepeso/obesidade, nos indivíduos com imagem corporal moderada/grave distorção e com risco de transtornos alimentares. Também, observou-se que a prevalência de imagem corporal moderada/grave distorção foi maior em adolescentes de 13 a 16 anos e com risco de transtornos alimentares. $\mathrm{O}$ presente estudo indicou que a maioria dos adolescentes dessa escola estava satisfeita com sua imagem corporal, apresentando eutrofia, demonstrando uma imagem positiva do próprio corpo. Porém, é necessária atenção nutricional àqueles com estado nutricional inadequado ou em risco de transtorno alimentar.
\end{abstract}

Palavras-chave: Avaliação nutricional. Adolescentes. Imagem corporal.

\section{Nutritional status and perception between teenage of the school city Guarapuava, PR}

\begin{abstract}
The objective of this study was to evaluate the nutritional status and body perception among adolescents in a school hall in the city of Guarapuava, PR. The study was conducted in a municipal school in Guarapuava, PR, attended by 39 adolescents of both genders, aged between 10 and 16 years. Weight and height were measured to assess nutritional status by body mass index (BMI), and later was applied questionnaire Body Shape Questionnaire (BSQ) for verification of body awareness. To assess the students' body satisfaction was used Silhouette Matching Task (SMT). The results showed that $79.5 \%$ of adolescents were eutrophic, $5.1 \%$ obese and $15.4 \%$ overweight. In general, individuals had a positive perception of their body image, however there was a prevalence of BMI and overweight/obesity, body image in individuals with moderate/severe distortion and at risk for eating disorders. Also, it was observed that the body image prevalence of moderate/severe distortion was greater in adolescents 13 to 16 years and at risk for eating disorders. This study indicated that most teenagers of that school were satisfied with their body image, with normal weight, demonstrating a positive body image. However, it is necessary nutritional care to those with inadequate nutritional status or at risk of eating disorder.
\end{abstract}

Keywords: Nutritional assessment. Adolescents. Body image.

\section{INTRODUÇÃO}

A adolescência é uma fase que ocorrem muitas transformações que podem ser tanto psicossociais, quanto nos hábitos alimentares

e estilo de vida o que pode acabar refletindo no estado nutricional dessa população (BRANCO et al., 2007). A adolescência é o 
período da vida que começa aos 10 anos de idade e estende-se até os 20 anos. Nesse período ocorre um elevado ritmo de crescimento e fenômenos maturativos importantes que afetam o tamanho, a forma e a composição do organismo (SOCIEDADE BRASILEIRA DE PEDIATRIA, 2012).

A imagem corporal é entendida como uma criação mental, de maneira em que se descrevem amplas representações internas da estrutura corporal e da aparência física do indivíduo, em relação a si próprio e aos outros (LIMA et al., 2008).

Ao longo do tempo, mudou-se o conceito de corpo saudável ou ideal, pois até o século XX considerava-se um corpo roliço como ideal, devido ao depósito de gordura nos quadris, coxas, barrigas e mamas (BOSI et al., 2006). Atualmente, a tendência social e cultural considera que o corpo ideal é um corpo magro. Portanto, o adolescente agrega em sua mente que deve chegar o mais próximo do corpo ideal, quanto mais ele se distanciar do real, maior será a possibilidade de diminuir a auto-estima desse indivíduo (BRANCO et al., 2006). A busca por esse corpo perfeito e outros fatores, acaba levando o adolescente a ter um distúrbio nutricional. Esse acontecimento acaba acarretando um período de risco nutricional, pois está associado à mudança nos hábitos alimentares, influência negativa dos amigos e, também, pressão da sociedade (FREITAS et al., 2009).
Dois dos distúrbios nutricionais comuns que os adolescentes podem desenvolver são a anorexia e a bulimia. A frequência desses distúrbios vem aumentando principalmente entre mulheres jovens, que pode ser ocasionado por: práticas inadequadas de controle de peso, insatisfação corporal e baixa auto-estima (NUNES et al., 2001). Sendo assim, o objetivo do presente estudo foi avaliar o estado nutricional, a percepção e satisfação corporal de adolescentes de uma escola municipal do município de Guarapuava, PR.

\section{MATERIAL E MÉTODOS}

\section{Local e população}

Trata-se de um estudo transversal, sendo realizado em uma escola municipal do município de Guarapuava, PR. Do total de 100 adolescentes matriculados na escola e contatados, apenas 39 aceitaram participar da pesquisa. A idade variou entre 10 a 16 anos, sendo de ambos os gêneros.

\section{Dados antropométricos}

Para a coleta dos dados, os alunos foram chamados em uma sala particular, individualmente, sendo aferidos o peso e estatura. Para a mensuração do peso utilizouse uma balança digital (Cadence Bal $150^{\circledR}$, Brasil) e para aferição da estatura foi utilizada uma fita métrica inelástica afixada em uma parede sem rodapé (ORGANIZAÇÃO 
MUNDIAL DE SAÚDE, 2007). O diagnóstico do estado nutricional foi realizado conforme o preconizado pelo Sistema de Vigilância Alimentar e Nutricional (SISVAN, 2004). Os resultados foram interpretados com o auxílio de Tabelas propostas pela Organização Mundial da Saúde (ORGANIZAÇÃO MUNDIAL DE SAÚDE, 2007), sendo avaliado o indicador IMC/idade, de acordo com a World Health Organization (1995).

Avaliação da percepção e satisfação corporal

Foi aplicado o questionário Body Shape Questionnaire (BSQ), não validado, que avalia a percepção corporal, segundo Cooper et al. (1987). O questionário foi adaptado e apresentava vinte e oito perguntas, as quais buscavam saber como os adolescentes sentiam-se em relação à sua aparência nas últimas quatro semanas. As respostas foram assinaladas de acordo com a legenda, onde 1: nunca; 2: raramente; 3: às vezes; 4: frequentemente; 5: muito frequentemente; 6: sempre. O BSQ foi distribuído nas turmas selecionadas, seguido de instruções básicas de respostas.

Para avaliar a satisfação corporal dos alunos foi utilizado o Silhouette Matching Task (SMT), proposto por Stunkard et al. (1983), adaptado por Marsh e Roche (1996). O SMT foi composto por 12 silhuetas em escala progressiva. Nesse instrumento de pesquisa o aluno marcava a silhueta a qual ele julgava mais parecida com a sua. O SMT foi distribuído nas turmas selecionadas, seguido de instruções básicas para preenchimento.

\section{Análise estatística}

Utilizou-se de análise estatística descritiva, além do teste de Qui-quadrado de Pearson. Foi realizado também, o teste de razão de prevalências (Odds Ratio Bruto OR) com intervalo de confiança (IC) de $95 \%$. O nível de significância foi fixado em $5 \%$ $(\mathrm{p}<0,05)$, sendo utilizado para os cálculos o software Statistical Package for Social Sciences $\left(\mathrm{SPSS}^{\circledR}\right)$ versão 19.0.

\section{Questões éticas}

Todas as normas da Resolução $\mathrm{n}^{\circ}$ 196/96 do Conselho Nacional de Saúde foram seguidas para realização dessa pesquisa. Anteriormente a coleta de dados, foi entregue a cada indivíduo um Termo de Consentimento Livre e Esclarecido (TCLE), sendo necessária a assinatura de seu responsável legal para a participação na pesquisa.

\section{RESULTADOS E DISCUSSÃO}

A amostra do estudo foi composta por $38,5 \%$ meninos e $61,5 \%$ por meninas. $\mathrm{Na}$ Tabela 1 apresentam-se dados descritivos relacionados à idade, IMC, peso, altura e silhueta dos adolescentes estudados. 
Tabela 1 - Dados descritivos relacionados à idade, IMC, peso, altura e silhueta dos adolescentes avaliados

\begin{tabular}{lcc}
\hline Avaliação & Média \pm DP & Máximo e Mínimo \\
\hline Idade (anos) & $12,33 \pm 1,48$ & $16-10$ \\
IMC (kg/m²) & $19,18 \pm 3,50$ & $14-33$ \\
Peso (kg) & $46,6 \pm 13,76$ & $23,8-98,4$ \\
Altura (m) & $1,54 \pm 0,11$ & $1,29-1,78$ \\
Silhuetas* & $4,31 \pm 2,36$ & $1-10$ \\
\hline Silhueta avaliada pelo Silhouette Matching Task (SMT) (MARSH; ROCHE, 1996); IMC: Índice de massa corporal; DP: Desvio padrão da média.
\end{tabular}

Analisando o IMC (Tabela 1) foi possível observar que em média os adolescentes apresentaram estado nutricional adequado (ORGANIZAÇÃO MUNDIAL DE SAÚDE, 2007). Com relação às silhuetas, observa-se que a classificação geral foi de "leve distorção", conforme o proposto por
Mash e Roche (1996) para ambos os gêneros, podendo ser classificada como eutrofia. Por meio da Tabela 2 verificam-se os resultados relacionados com o IMC, imagem corporal e o risco dos adolescentes apresentarem transtorno ou não, separado por gêneros (feminino e masculino).

Tabela 2 - Dados relacionados ao IMC, imagem corporal e risco de transtorno em relação aos gêneros femininos e masculinos

\begin{tabular}{|c|c|c|c|c|}
\hline \multirow[t]{2}{*}{ Avaliações } & \multicolumn{2}{|c|}{ Feminino } & \multicolumn{2}{|c|}{ Masculino } \\
\hline & $\mathrm{n}$ & $\%$ & $\mathrm{n}$ & $\%$ \\
\hline \multicolumn{5}{|l|}{ IMC } \\
\hline Eutrofia & 21 & 87,50 & 10 & 66,66 \\
\hline Sobrepeso & 2 & 8,33 & 4 & 26,66 \\
\hline Obesidade & 1 & 4,16 & 1 & 6,66 \\
\hline \multicolumn{5}{|c|}{ Imagem Corporal (BSQ) } \\
\hline Normalidade & 15 & 62,50 & 12 & 80,00 \\
\hline Leve distorção & 1 & 4,16 & 1 & 6,66 \\
\hline Moderada distorção & 5 & 20,83 & 1 & 6,66 \\
\hline Grave distorção & 3 & 12,50 & 1 & 6,66 \\
\hline \multicolumn{5}{|c|}{ Risco de transtorno alimentar } \\
\hline Sem risco & 19 & 79,20 & 10 & 66,70 \\
\hline Risco & 5 & 20,80 & 5 & 33,30 \\
\hline
\end{tabular}

Os resultados do IMC se mostraram favorável para ambos os gêneros, uma vez que a maioria das meninas e dos meninos encontrava-se eutróficos. Os percentuais de sobrepeso e obesidade foram baixos entre toda a população estudada.

Com relação à imagem corporal e risco de transtornos alimentares, a maioria das meninas e dos meninos apresentou resultados da silhueta adequados, dentro da normalidade, sem risco de transtorno. Porém, a pequena porcentagem de ambos os gêneros, que se mostraram em risco, pode ser explicada devido ao fato de se tratar de adolescentes, que em geral sempre estão preocupados com o corpo, muitas vezes buscando padrões que a sociedade julga adequado, apresentando assim, distorções com relação à silhueta e risco de transtorno alimentar. Na Tabela 3 apresentam-se alguns dados relacionados às perguntas presentes no questionário BSQ sobre percepção corporal respondido pela população do estudo. 
Tabela 3 - Dados relacionados às perguntas contidas no Body Shape Questionnaire (BSQ) aplicado aos adolescentes avaliados

\begin{tabular}{|c|c|c|c|c|c|c|}
\hline $\begin{array}{l}\text { Respostas/ } \\
\text { Perguntas } \\
\end{array}$ & $\mathbf{A V}$ & $\mathbf{F}$ & MF & $\mathbf{N}$ & $\mathbf{R}$ & $\mathbf{S}$ \\
\hline \multicolumn{7}{|c|}{ Sentir-se mal com o corpo a ponto de chorar? } \\
\hline $\mathrm{n}$ & 4 & 2 & 1 & 27 & 3 & 2 \\
\hline$\%$ & 10,3 & 5,1 & 2,6 & 69,2 & 7,7 & 5,1 \\
\hline \multicolumn{7}{|c|}{ Sentir-se gorda após ingerir uma pequena refeição? } \\
\hline $\mathrm{n}$ & 3 & 1 & 1 & 25 & 5 & 4 \\
\hline$\%$ & 7,7 & 2,6 & 2,6 & 64,1 & 12,8 & 10,3 \\
\hline \multicolumn{7}{|c|}{ Preocupação em ocupar muito espaço quando está acompanhado? } \\
\hline $\mathrm{n}$ & 2 & 2 & - & 31 & 3 & 1 \\
\hline$\%$ & 5,1 & 5,1 & - & 79,5 & 7,7 & 2,6 \\
\hline \multicolumn{7}{|c|}{ Você belisca áreas de seu corpo para ver o quanto há de gordura? } \\
\hline $\mathrm{n}$ & 4 & 1 & 1 & 24 & 7 & 2 \\
\hline$\%$ & 10,3 & 2,6 & 2,6 & 61,5 & 17,9 & 5,1 \\
\hline
\end{tabular}

A prevalência de insatisfação com a imagem corporal encontrada neste estudo foi positiva, pois a maioria dos adolescentes se mostrou pouco preocupados com seu corpo, apresentando respostas como "nunca" ou "raramente", para perguntas relacionadas ao fato de seu corpo não ser suficientemente firme, se sentir preocupada em relação ao seu físico perto de pessoas magras e evitar usar roupas que fazem notar as formas do seu corpo.

Algumas perguntas feitas aos adolescentes obtiveram-se mais respostas "nunca e raramente" (Tabela 3), dente elas estavam: já se sentiu mal com o corpo a ponto de chorar $(76,9 \%, \mathrm{n}=30)$; se sentir gorda após ingerir uma pequena refeição, sentir vergonha do próprio corpo $(71,8 \%, \mathrm{n}=28)$, evitar correr pelo fato de que seu corpo poderia balançar $(89,8 \%, n=35)$, pensar no seu físico interfere em sua capacidade de se concentrar em outras atividades $(71,7 \%, \mathrm{n}=28)$, deixar de participar de eventos sociais por sentir-se mal em relação ao seu físico, preocupação em ocupar muito espaço quando está acompanhado $(87,2 \%, n=34)$, beliscar áreas de seu corpo para ver o quanto há de gordura $(79,4 \%$, $\mathrm{n}=31$ ). Na Tabela 4 encontram-se os dados de prevalência de IMC com sobrepeso/ obesidade, segundo gênero, idade, dados do questionário BSQ e relacionados ao risco de transtorno alimentar. 
Tabela 4 - Prevalência de IMC com sobrepeso/obesidade, segundo gênero, idade, imagem corporal e risco de transtorno alimentar dos adolescentes avaliados

\begin{tabular}{|c|c|c|c|c|c|c|}
\hline & \multirow[b]{2}{*}{$\mathrm{n}$ total } & \multicolumn{5}{|c|}{ Prevalência de IMC sobrepeso/obesidade } \\
\hline & & $\mathrm{n}$ & $\%$ & $\mathrm{p}^{*}$ & OR bruto (IC95\%) & $\mathrm{p}^{* *}$ \\
\hline Gênero & & & & 0,69 & & \\
\hline Feminino & 24 & 4 & 16,7 & & - & - \\
\hline Masculino & 15 & 4 & 26,7 & & - & - \\
\hline Idade & & & & 0,26 & & \\
\hline $10-12$ & 22 & 3 & 37,5 & & - & - \\
\hline $13-16$ & 17 & 5 & 62,5 & & - & - \\
\hline Imagem Corporal (BSQ) & & & & 0,02 & & \\
\hline Normalidade/leve distorção & 29 & 3 & 37,5 & & 1 & 0,01 \\
\hline Moderada/grave distorção & 10 & 5 & 62,5 & & $8,67(1,55-48,49)$ & \\
\hline Risco de transtorno alimentar & & & & 0,02 & & \\
\hline Sem risco & 29 & 3 & 37,5 & & 1 & 0,01 \\
\hline Risco & 10 & 5 & 62,5 & & $8,67(1,55-48,49)$ & \\
\hline
\end{tabular}

Não houve prevalência de IMC com sobrepeso/obesidade avaliando-se os gêneros e idade dos adolescentes (Tabela 4). Entretanto, verifica-se maior prevalência de IMC com sobrepeso/ obesidade nos adolescentes que apresentaram imagem corporal com moderada/ grave distorção e com risco de transtornos alimentares $(\mathrm{p}<0,05)$. Já, a análise de regressão logística
(OR) mostrou que os indivíduos que apresentam IMC de sobrepeso/obesidade têm 8,67 mais chances de ter $(\mathrm{p}<0,05)$ imagem corporal com moderada/ grave distorção e risco de transtornos alimentares. Na Tabela 5 é observa-se a prevalência de imagem corporal com moderada/ grave distorção relacionada com gênero, idade e risco de transtorno alimentar.

Tabela 5 - Prevalência de imagem corporal com moderada/grave distorção e total da população estudada divididos em gênero, idade e risco de transtorno alimentar dos adolescentes avaliados

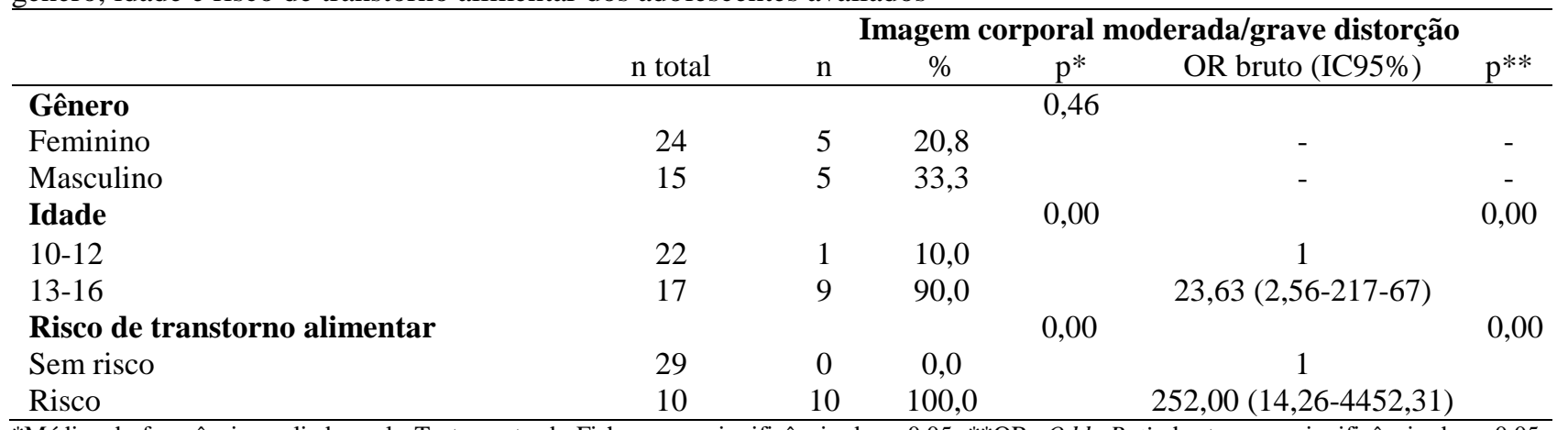

*Médias de frequência avaliadas pelo Teste exato de Fisher, com significância de p<0,05. **OR: Odds Ratio bruto, com significância de p<0,05; BSQ: Body Shape Questionnaire (COOPER et al., 1987).

Não houve prevalência de imagem corporal com moderada/grave distorção entre os gêneros (Tabela 5), entretanto observou-se maior predomínio de imagem corporal com moderada/grave distorção nos adolescentes entre 13-16 anos e naqueles com risco de transtornos alimentares.

A razão de chances apontou que os indivíduos de 13 a 16 anos têm 23,62 vezes mais chances de ter $(\mathrm{p}<0,05)$ silhueta 
moderada/ grave do que aqueles com 10 a 12 anos, e, também que os adolescentes com risco de transtorno alimentar possuem 252,00 vezes mais chances de apresentar prevalência de imagem corporal com moderada/grave distorção.

No presente estudo a maioria da população é composta do gênero feminino $(61,5 \%)$, diferente do trabalho de Albano e Souza (2001) que encontraram 44,9\% do gênero feminino e $53,5 \%$ do gênero masculino. Isso pode ser explicado devido ao fato de que muitos meninos se recusaram a participar do estudo. Em relação ao resultado geral da avaliação do IMC da população estudada, $\quad 79,48 \% \quad(\mathrm{n}=31) \quad$ apresentaram eutrofia e 5,12\% (n=2) obesidade esses resultados corroboram com Silva et al. (2008), que encontraram em seu estudo $70,4 \%$ de adolescentes eutróficos e 3,7\% com obesidade.

Os adolescentes foram questionados, também, sobre o uso de laxantes e prática de vômitos, sendo que $94,9 \%$ nunca utilizaram estes métodos para se sentir mais magros. Este resultado é positivo, uma vez que a população se mostra menos susceptível a desenvolver algum tipo de transtorno alimentar. Conforme explica Cordás (2004), a preocupação excessiva com o peso e a imagem corporal leva o adolescente a métodos compensatórios inadequados para o controle de peso como vômitos auto- induzidos, uso de medicamentos, como diuréticos e laxantes.

Na Tabela 4 é possível observar que há uma pequena insatisfação corporal por parte dos adolescentes, assim como verificado em trabalhos de Aertes et al. (2010). Entretanto, Vilela et al. (2004) que utilizaram o Teste de Atitudes Alimentares, Teste de Investigação Bulímica de Edinburgh e Teste de Imagem Corporal para classificar a satisfação corporal em escolares de cinco localidades do interior de Minas Gerais, encontraram 59\% de alunos insatisfeitos com seu físico.

Analisando os resultados obtidos por meio do questionário BSQ (Tabela 5) observou-se que a maioria das respostas para a questão foi "nunca" e "raramente", mostrando assim que a maioria dos adolescentes não indicou insatisfação corporal. Ressalta-se que como $79,48 \%$ dos adolescentes apresentaram eutrofia, os mesmos provavelmente possam ter uma percepção de sua imagem corporal mais positiva, não apresentando graves distorções. Porém, estudo realizado por Freitas et al. (2009) constatou altos índices de insatisfação corporal entre adolescentes, onde apenas $29,63 \%$ das participantes referiram estar contentes com seu corpo.

Conforme Branco et al. (2006) a imagem corporal é a capacidade de representação mental do próprio corpo pertinente a cada indivíduo, sendo que essa imagem envolve aspectos relacionados à 
estrutura (como tamanho, dimensões) e à aparência (forma, aspecto), entre vários outros componentes psicológicos e físicos da imagem corporal.

No presente estudo, verifica-se na Tabela 2 que entre os adolescentes que apresentaram "leves" e "graves" distorções, $16,66 \%$ eram meninas e $13,32 \%$ eram meninos. Segundo explicam Ricciardelli et al. (2000), as influências socioculturais afetam diferentemente meninos e meninas. Enquanto meninos são estimulados a praticarem atividades esportivas, meninas são estimuladas a praticarem atividades que impliquem em perda de peso. Conti et al. (2005) observaram em estudo com adolescentes que meninas recebem reforço para cuidar-se e possuem preocupação com o caráter estético, enquanto meninos são mais focados no aspecto lúdico e relacional. Esta diferença social, possivelmente, oferece aos jovens a aquisição de padrões estéticos e pessoais específicos, com as meninas tendendo a estar mais insatisfeita quando comparadas aos meninos. O'dea (2001) e Brodie et al. (2004) indicam que a idade é um importante fator, sendo necessário controlá-lo nas pesquisas envolvendo imagem corporal. Brodie et al. (2004), afirma que o fenômeno "imagem corporal" não é totalmente compreendido, entretanto é necessário aproximá-lo de diferentes aspectos, o que incluiria o psicológico, psiquiátrico, somático, neurológico entre outros.

\section{CONCLUSÃO}

A maioria dos adolescentes avaliados estava satisfeita com sua imagem corporal, sem alterações no estado nutricional e, assim, demonstram uma imagem positiva do próprio corpo. Entretanto, é preciso atenção especial à outra parte de adolescentes que se mostraram insatisfeitos. Pois, é essa a fase da vida que requer um maior cuidado com questões relacionadas ao corpo, uma vez que é na adolescência que ocorre às diversas transformações, tanto psicossociais como no estilo de vida e hábitos alimentares.

$\mathrm{O}$ estado nutricional de eutrofia se destacou nos adolescentes em geral, sendo que a maioria apresenta-se satisfeito com o corpo, e sem risco de desenvolver transtornos alimentares. Esses fatores podem ser considerados pontos positivos para essa escola, pois essas questões não afetaram o aprendizado dos alunos. Entretanto, ressaltase que na população avaliada a presença de moderada/grave distorção e risco de transtorno alimentar elevou a prevalência de IMC sobrepeso/obesidade. A idade entre 13 a 16 anos e a presença de risco de transtorno alimentar, também aumentaram a prevalência de imagem corporal moderada/grave distorção.

A escola, como um local privilegiado para oferecer informações sobre estilos de vida mais saudáveis, deve estar atenta a esse problema e, juntamente com os serviços de 
saúde, definir estratégias de atuação reduzindo estes problemas.

\section{REFERÊNCIAS}

AERTES, D.; MADEIRA, R.R.; ZART, V.B. Imagem corporal de adolescentes escolares em Gravataí-RS. Epidemiologia e Serviços de Saúde, v.19, n.3, p.283-91, 2010.

ALBANO, R.D.; SOUZA, S.B. Estado nutricional de adolescentes: "risco de sobrepeso" e "sobrepeso" em uma escola pública do Município de São Paulo.

Cadernos de Saúde Pública, v.17, n.4, p.941-7, 2001.

BOSI, M.L.M.; LUIZ, R.R.; MORGADO, C.M.C.; COSTA, M.L.S.; CARVALHO, R.J. Autopercepção da imagem corporal entre estudantes de nutrição: um estudo no município do Rio de Janeiro. Jornal Brasileiro de Psiquiatria, v.55, n.2, p.10813, 2006.

BRANCO, L.M.; ALMEIDA, E.C.; PASSOS, M.A.Z.; PIANO, A.; CINTRA, I.P. A percepção corporal influencia no consumo do café da manhã de adolescentes? Saúde em Revista, v.9, n.22, p.15-21, 2007.

BRANCO, L.M.; HILÁRIO, M.O.E.; CINTRA, I.P. Percepção e satisfação corporal em adolescentes e a relação com seu estado nutricional. Revista de Psiquiatria Clínica, v.6, n.33, p.292-6, 2006.

BRODIE, D.A.; BAGLEY, K.; SLADE, P.D. Body image perception in pre-and post adolescent females. Perceptual and Motor Skills, v.78, n.1, p.147-54, 1994.

CONTI, M.A.; GAMBARDELLA, A.D.; FRUTUOSO, F.M.P. Insatisfação com a imagem corporal em adolescentes e sua relação com a maturação sexual. Revista Brasileira de Crescimento e Desenvolvimento Humano, v.15, n.2, p.3644, 2005.
COOPER, P.J.; TAYLOR, M.J.; COOPER, Z. The development and validation of the body shape questionnaire. International Journal of Eating Disorders, v.6, n.4, p.485-94, 1987.

CORDAS, T.A. Transtornos alimentares: classificação e diagnóstico. Revista de Psiquiatria Clínica, v.31, n.4, p.154-7, 2004.

FREITAS, A.R.; NOVELLO, D.; GASTALDON, L.T.; JUSTINO, P.F. Insatisfação da imagem corporal, práticas alimentares e de emagrecimento em adolescentes do sexo feminino. Revista Brasileira de Nutrição Clínica, v.24, n.3, p.166-73, 2009.

LIMA, J.R.P.; ORLANDO, F.B.; TEIXEIRA, M.P.; CASTRO, A.P.A.; DAMASCENO, V.O. Conjunto de silhuetas para avaliar a imagem corporal de participantes de musculação. Arquivos Sanny de Pesquisa em Saúde, v.1, n.1, p.26-30, 2008.

MARSH, H.W.; ROCHE, L.A. Predicting self-esteem from perceptions of actual and ideal ratings of body fatness: is there only one ideal "supermodel". Research Quarterly for Exercise and Sport, v.67, n.1, p.3-23, 1996.

NUNES, M.A.; OLINTO, M.T.A.; BARROSA, F.C.; CAMEYC, S. Influência da percepção corporal do peso e do índice de massa corporal nos comportamentos alimentares anormais. Revista Brasileira de Psiquiatria, v.23, n.1, p.21-7, 2001.

O'DEA, J.C.P. Association between socioeconomic status, weight, age and gender, and the body image and weight control practices of 6- to 19-year-old children and adolescent. Health Education Research, v.16, n.5, p.521-32, 2001.

ORGANIZAÇÃO MUNDIAL DE SAÚDE OMS. Curvas de crescimento. Brasília, DF: SISVAN, 2007.

RICCIARDELLI, L.A.; MCCABE, M.P.; BANFIELD, S. Body image and body change methods in adolescent boys role of parents, 
friends and the media. Journal of Psychatric

Research, v.49, n.3, p.189-97, 2000.

SILVA, G.R.; CRUZ, N.R.; COELHO, E.J.B. Perfil nutricional, consumo alimentar e prevalência de sintomas de anorexia e bulimia nervosa em adolescentes de uma escola da rede pública no município de Ipatinga, MG. Revista Digital de Nutrição Unileste, v.2, n.3, p.1-15, 2008.

SISTEMA DE VIGILÂNCIA ALIMENTAR E NUTRICIONAL - SISVAN. SISVAN -

Orientações básicas para a coleta, processamento, análise de dados e a informação em serviços de saúde. Brasília, DF: SISVAN, 2004.

\section{SOCIEDADE BRASILEIRA DE}

PEDIATRIA. Manual de orientação para alimentação do lactente, do pré-escolar, do escolar, do adolescente e na escola.

Sociedade Brasileira de Pediatria.

Departamento de Nutrologia. $3^{\mathrm{a}}$. ed. Rio de Janeiro, RJ: SBP, 2012.

STUNKARD, A.J.; SORENSON, T.; SCHULSINGER, F. Use of the Danish Adaption Registry for the study of obesity and thinness. Research Publications Association for Research in Nervous and Mental Disease, v.60, n.1, p.115-20, 1983.

VILELA， J.E.M.; LAMOUNIER， J.A.; DELLARETTI FILHO, M.A.; BARROS JR, N.; HORTA, G.M. Transtornos alimentares em escolares. Jornal de Pediatria, v.80, n.1, p.49-54, 2004.

WORLD HEALTH ORGANIZATION WHO. Infants and children. In: World Health Organization Physical status: the use and interpretation of anthropometry. WHO, v.85, p.111-75, 1995. 